\title{
Exciton Dephasing in Quantum Dots due to LO-Phonon Coupling: An Exactly Solvable Model
}

\author{
E. A. Muljarov ${ }^{1,2, *}$ and R. Zimmermann ${ }^{1}$ \\ ${ }^{1}$ Institut für Physik der Humboldt-Universität zu Berlin, Newtonstrasse 15, D-12489 Berlin, Germany \\ ${ }^{2}$ General Physics Institute, Russian Academy of Sciences, Vavilova 38, Moscow 119991, Russia
}

(Received 23 May 2006; published 1 May 2007)

\begin{abstract}
It is widely believed that, due to its discrete nature, excitonic states in a quantum dot coupled to dispersionless longitudinal-optical (LO) phonons form everlasting mixed states (exciton polarons) showing no line broadening in the spectrum. This is indeed true if the model is restricted to a limited number of excitonic states in a quantum dot. We show, however, that extending the model to a large number of states results in LO phonon-induced spectral broadening and complete decoherence of the optical response.
\end{abstract}

DOI: 10.1103/PhysRevLett.98.187401

Among different mechanisms of the optical decoherence in semiconductor quantum dots (QDs), the interaction between excitons and lattice vibrations (phonons) is the most important one at low carrier densities, leading to a temperature-dependent linewidth in optical spectra. The discrete nature of excitonic levels in a QD gave rise to the so-called phonon bottleneck problem [1]: When the phonon energy does not fit the level separation, real phonon-assisted transitions between different excitonic states are not allowed. On the other hand, the measured optical polarization in QDs shows a partial initial decoherence and a temperature-dependent exponential decay at larger times [2]. The bottleneck problem is partly removed for acoustic phonons as they have an energy dispersion and thus contribute to carrier relaxation [3]. Moreover, even apart from the resonance between the phonon energy and the level separation, acoustic phonons, due to their dispersion, are shown to be responsible for the long-time exponential decay induced by virtual processes [4].

Longitudinal-optical (LO) phonons, in turn, have a negligible dispersion which leads to a quite different behavior: Excitons in QDs and LO phonons are always in a strong coupling regime [5] in the sense that they form everlasting polarons with no spectral broadening [6-9]. This also makes any approximate perturbative approach to the exciton-LO phonon problem inappropriate. Indeed, for a few excitonic levels in a QD coupled to bulk LO phonons, a self-consistent second Born approximation for the selfenergy developed in $[10,11]$ results in a line broadening which is fully artificial. This artefact is refuted by the exact solution of this problem [6,9] showing that the spectrum consists exclusively of discrete unbroadened lines. Also, a Gauss spectral line shape was found in the quadratic coupling model by truncating the cumulant expansion in second order [12]. Again, the exact solution of this model shows no spectral broadening [13].

In this Letter we concentrate on the long-time behavior of the optical polarization and show that, even without acoustic phonon contribution, pronounced phononinduced dephasing and spectral broadening do nevertheless exist in QDs. This broadening is calculated microscopi-
PACS numbers: 78.67.Hc, 03.65.Yz, 71.35.-y, 71.38.-k

cally by inclusion of infinitely many excitonic states in a QD that has never been done before. Thus a qualitatively new source of the dephasing in QDs is found.

The full problem of excitons in a QD linearly coupled to the LO phonon displacement can be solved exactly only for a very limited number of excitonic states [9]. The major obstacles are off-diagonal terms in the exciton-phonon interaction which couple different excitonic states in a QD. In order to take into account as many excitonic states as we like, we have derived microscopically an effective exciton-phonon Hamiltonian which has only leveldiagonal terms and thus allows an exact solution for an arbitrary number of states. This effective Hamiltonian, however, preserves the main features of the original problem, since the off-diagonal terms are also intrinsically represented: By means of a unitary transformation they are mapped into diagonal terms giving rise to an excitonphonon coupling quadratic in the phonon displacement operators $[4,14]$.

Concentrating on the ground exciton state $|1\rangle$, the effective Hamiltonian takes the form $(\hbar=1)$ :

$$
\begin{gathered}
H=\omega_{0} \sum_{\mathbf{q}} a_{\mathbf{q}}^{\dagger} a_{\mathbf{q}}+\left(E_{1}+V_{L}+V_{Q}\right)|1\rangle\langle 1|, \\
V_{L}=\sum_{\mathbf{q}} M_{11}(\mathbf{q})\left(a_{\mathbf{q}}+a_{-\mathbf{q}}^{\dagger}\right), \\
V_{Q}=-\frac{1}{2} \sum_{\mathbf{p q}} Q(\mathbf{p}, \mathbf{q})\left(a_{\mathbf{p}}+a_{-\mathbf{p}}^{\dagger}\right)\left(a_{\mathbf{q}}+a_{-\mathbf{q}}^{\dagger}\right), \\
Q(\mathbf{p}, \mathbf{q})=2 \sum_{n \neq 1} \frac{E_{n}-E_{1}}{\left(E_{n}-E_{1}\right)^{2}-\omega_{0}^{2}} M_{1 n}(\mathbf{p}) M_{n 1}(\mathbf{q}) \\
\equiv \sum_{n \neq 1} F_{n}(\mathbf{p}) F_{n}(\mathbf{q}),
\end{gathered}
$$

where $\omega_{0}$ is the dispersionless LO phonon frequency and $E_{n}$ is the bare transition energy of a single-exciton state $|n\rangle$ in a $\mathrm{QD}$. The kernel $Q$ of the quadratic coupling to LO phonons has the same form as the effective scattering matrix used in Ref. [14] to describe the phonon modes bound to a neutral donor. It is derived from the level- 
nondiagonal matrix elements $M_{1 n}(\mathbf{q}) \propto q^{-1}\langle 1| e^{i \mathbf{q r} \mathbf{r}_{e}}-$ $e^{i \mathbf{q} \mathbf{r}_{h}}|n\rangle$ of the linear exciton-phonon (Fröhlich) interaction, up to second order, and expressed in Eq. (4) in terms of functions $F_{n}$. A quadratic coupling model similar to Eqs. (1)-(4) has already been successfully used for calculation of the exciton dephasing in InGaAs QDs, induced by acoustic phonon-assisted virtual transitions [4].

The method developed in [4] allows us to find the exact solution of the Hamiltonian (1)-(4), using the cumulant expansion. The linear optical polarization is given by $\int_{-\infty}^{t} \mathcal{E}(\tau) P(t-\tau) d \tau$, where $\mathcal{E}(t)$ is the electric field of the driving optical pulse,

$$
P(t)=\theta(t) \exp \left[-i E_{1} t+K_{L}(t)+K_{Q}(t)\right],
$$

and the linear and quadratic cumulants are

$$
\begin{aligned}
K_{L}(t)= & {\left[(2 N+1)\left(\cos \omega_{0} t-1\right)+i\left(\omega_{0} t-\sin \omega_{0} t\right)\right] } \\
& \times \sum_{\mathbf{q}}\left|M_{11}(\mathbf{q}) / \omega_{0}\right|^{2} \\
K_{Q}(t)= & -\frac{1}{2} \sum_{\nu j} \ln \left[1-i \Lambda_{\nu} \lambda_{j}(t)\right] .
\end{aligned}
$$

Here $\Lambda_{\nu}$ are the eigenvalues of the matrix

$$
A_{n m}=\sum_{\mathbf{q}} F_{n}(-\mathbf{q}) F_{m}(\mathbf{q}),
$$

while $\lambda_{j}(t)$ are the eigenvalues of the Fredholm problem

$$
\int_{0}^{t} d \tau^{\prime} D\left(\tau-\tau^{\prime}\right) u_{j}\left(\tau^{\prime} ; t\right)=\lambda_{j}(t) u_{j}(\tau ; t)
$$

Equation (9) can be solved numerically, as it has been done for acoustic phonons [4]. However, for dispersionless optical phonons the propagator $D(t) \equiv\left\langle\mathcal{T}\left[a_{\mathbf{q}}(t) a_{\mathbf{q}}^{\dagger}+\right.\right.$ $\left.\left.a_{\mathbf{q}}^{\dagger}(t) a_{\mathbf{q}}\right]\right\rangle$ is $\mathbf{q}$ independent: $D(t)=(N+1) e^{-i \omega_{0}|t|}+$ $N e^{i \omega_{0}|t|}$ [where $N=1 /\left(e^{\beta \omega_{0}}-1\right)$ and $\beta=1 / k_{B} T$ ] and thus allows an analytic solution of Eq. (9) which can be found in Ref. [13]. Strictly speaking, the mixed cumulant $K_{M}(t)$ introduced in Ref. [4] would also appear in Eq. (5); however, it leads to only tiny corrections: $\left|K_{M} / K_{Q}\right|<$ $10^{-7}$ in CdSe QDs considered here.

We have already used the model Eqs. (1)-(4) and its solution Eqs. (5)-(9) in the case of two excitonic levels in an InGaAs QD [13] to show that a truncation of an infinite series in the cumulant as done in Ref. [12] leads to an artificial Gauss decay of the optical polarization. Only taking into account an infinite number of all-order diagrams gives the correct result which is an almost perfectly periodic time-dependent polarization. In the present Letter, using the same model, we include now (infinitely) many excitonic states in a QD and show that this leads to a qualitatively new effect: polarization decay and spectral broadening.

This effect takes place in any type of semiconductor QDs. For the sake of illustration, we concentrate on the polar material CdSe and a simple model of a QD. Material parameters (Fröhlich coupling constants $\alpha_{e}=0.47, \alpha_{h}=$ $0.88 ; \hbar \omega_{0}=24 \mathrm{meV}$ ) and the QD model are the same as in
Ref. [9], but the parabolic confinement potentials are taken as isotropic. For the level distances $\Delta_{e, h}$ and Gauss localization lengths $l_{e, h}$ of electron and hole we use here three sets of parameters: (A) $\Delta_{e}=80 \mathrm{meV}, \Delta_{h}=35 \mathrm{meV}$, $l_{e}=l_{h}=2.5 \mathrm{~nm}$; (B) $\Delta_{e, h}$ are the same as in set (A), $l_{e}=$ $2.7 \mathrm{~nm}, l_{h}=2.2 \mathrm{~nm}$ (as in Ref. [9]); and (C) $\Delta_{e}=$ $40 \mathrm{meV}, \Delta_{h}=17.5 \mathrm{meV}, l_{e}=4.14 \mathrm{~nm}, l_{h}=3.36 \mathrm{~nm}$. We decided to neglect the Coulomb interaction as it would only renormalize the transition energies $E_{n}$ and induce minor changes in the polarization dynamics of the ground exciton state.

The amplitude of the time-dependent linear polarization after a Gauss-shaped excitation pulse (with duration of $200 \mathrm{fs}$ ) in a CdSe QD at $T=150 \mathrm{~K}$ is shown in Fig. 1 for set (A). In this case, due to the full charge neutrality of the electron-hole pair, $M_{11}(\mathbf{q})=0$ holds, and the linear term $V_{L}$ vanishes. Thus we concentrate at the moment on the quadratic term $V_{Q}$, Eq. (3), which is in fact the only source of dephasing. For two excitonic levels [i.e., only one excited state $n=2$ contributes to Eq. (4), and the matrix $A_{n m}$, Eq. (8), reduces to a scalar] the polarization has only tiny oscillations close to unity. For five levels, it decays and then revives almost to unity after 20 ps. For 30 levels, the amplitude decays already dramatically and then oscillates irregularly around a small value. Such a behavior has been discussed before and called quasidephasing $[9,15]$. Finally, if we take into account all excitonic states, the polarization decays strictly: At $100 \mathrm{ps}$ its amplitude drops to $10^{-30}$ (but is shown in Fig. 1 only up to $10^{-8}$ ). In practice, of course, we truncate the infinite matrix $A_{n m}$ and check that a larger matrix produces no changes within the time span considered.

To understand better why the optical polarization becomes decaying when more and more exciton (electronhole pair) states are taken into account, we calculate the linear absorption spectrum, i.e., the real part of the Fourier transform of $P(t)$. It is hopeless, however, to find the Fourier transform numerically if the function does not

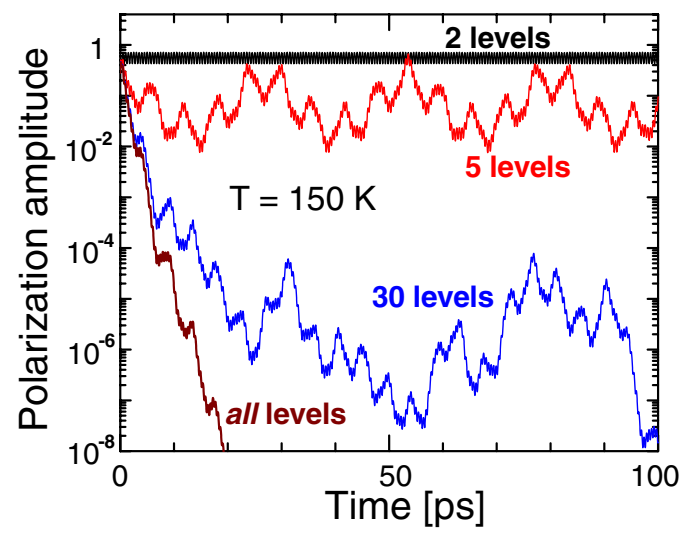

FIG. 1 (color online). Amplitude of the linear optical polarization of the ground exciton state at $T=150 \mathrm{~K}$, taking into account two, five, thirty, and all exciton levels in a CdSe QD (set A). 
decay at all or decays very weakly. That is why we use a different method here: We diagonalize exactly the excited state Hamiltonian $H_{1}$ which is related to the full one, Eq. (1), as $H=H_{0}|0\rangle\left\langle 0\left|+H_{1}\right| 1\right\rangle\langle 1|$. Such a diagonalization is very easy if $V_{L}=0$. In the case of two levels $H_{1}$ becomes

$$
H_{1}^{(2 \text { lev })}=\Omega B^{\dagger} B+\tilde{E}_{1}+\omega_{0} \sum_{\mu} b_{\mu}^{\dagger} b_{\mu},
$$

where

$$
\begin{aligned}
\Omega & =\sqrt{\omega_{0}^{2}-2 \omega_{0} \sum_{\mathbf{q}} F_{2}(-\mathbf{q}) F_{2}(\mathbf{q})}, \\
B & =\sum_{\mathbf{q}} F_{2}(\mathbf{q})\left(\xi_{+} a_{\mathbf{q}}+\xi_{-} a_{-\mathbf{q}}^{\dagger}\right)
\end{aligned}
$$

are, respectively, the new phonon frequency and annihila-

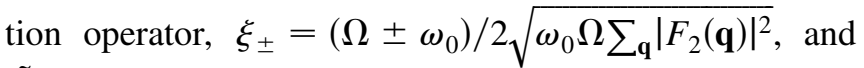
$\tilde{E}_{1}$ is the polaron shifted transition energy.

Note that out of a continuum of degenerate LO modes only a single phonon mode couples to the $\mathrm{QD}$ and produces a new, bound mode [14] $B$ with frequency $\Omega$, while all the other modes $b_{\mu}$, which show up in the last term of Eq. (10), are decoupled. They are simply some orthogonal linear combinations of the former phonon modes $a_{\mathbf{q}}$ and have the same old frequency $\omega_{0}$. The linear polarization $P(t)=$ $\theta(t)\left\langle e^{i H_{0} t} e^{-i H_{1} t}\right\rangle$, calculated in the same way as in Ref. [9], then takes the form

$$
P^{(2 \text { lev })}(t)=\theta(t) \sum_{\mathbf{n}, \mathbf{m}=0}^{\infty} \alpha_{\mathbf{n m}} \exp \left\{i\left(\mathbf{n} \omega_{0}-\mathbf{m} \Omega\right) t\right\},
$$

where $\alpha_{\mathbf{n m}}=\left.\left(1-e^{-\beta \omega_{0}}\right) e^{-\beta \mathbf{n} \omega_{0}}|\langle\mathbf{n}| \mathbf{m})\right|^{2}$, and $\left.\langle\mathbf{n}| \mathbf{m}\right)$ are the projections of new phonon states $\mid \mathbf{m})$ into old ones $|\mathbf{n}\rangle$ (to be distinguished from exciton states $|n\rangle$ ), which are calculated recursively.

Generalization to $\mathcal{N}$ excitonic levels is straightforward: $H_{1}$ is now diagonalized to $\mathcal{N}-1$ new phonon modes,

$$
\Omega_{\nu}=\sqrt{\omega_{0}^{2}-2 \omega_{0} \Lambda_{\nu}}
$$

with $\Lambda_{\nu}$ being the eigenvalues of the $(\mathcal{N}-1)$ dimensional matrix Eq. (8). In accordance with Eqs. (5) and (7), the full linear polarization can be written as a product

$$
P(t)=\prod_{\nu} P_{\nu}(t)
$$

of functions $P_{\nu}(t)$ due to each individual phonon mode given by the same Eq. (13), where $\Omega$ and $\alpha_{\mathbf{n m}}$ are replaced, respectively, by $\Omega_{\nu}$ and $\alpha_{\mathbf{n m}}^{\nu}$.

For two levels, the absorption spectrum, already discussed in Ref. [13], represents a set of discrete lines. There are two-phonon satellites around the zero-phonon line (the standard one-phonon satellites are absent here as $V_{L}=0$ ), but more important is the fine structure shown in
Fig. 2(a). This fine structure is due to the difference between the old and the new phonon frequency $\omega_{0}-\Omega$ and manifests itself in the time domain as rapid oscillations with the period of $0.5 \mathrm{ps}$ (Fig. 1). Including the third excitonic level brings in an additional frequency and more lines in the spectrum; Fig. 2(b). With five levels (and four new phonon frequencies), there is already plenty of closely lying delta lines; Fig. 2(c). Finally, if we include all exciton levels, these lines merge and produce a continuous broadening; Fig. 2(d). In the time domain, the polarization represents a superposition of individual oscillations [see Eqs. (13) and (15)] which interfere destructively due to incommensurability of their frequencies and thus lead to dephasing.

In contrast to the model of a QD with discrete exciton levels only, in realistic QD systems there is also a wettinglayer continuum. This continuum could seem to be a more probable candidate for producing the spectral broadening [16]. It turns out, however, that in deep QDs, there are usually enough discrete exciton levels to provide an appreciable polarization decay. In fact, taking into account 30 levels [17] is already sufficient to have the dephasing time very close to the exact one (i.e., with all discrete levels in harmonic potentials; see Fig. 1). In shallow QDs, there might be only one discrete exciton level, while all other states are delocalized in the wetting layer. The spectrum of such a QD, with the same harmonic potentials replaced for $r_{e, h}>5 \mathrm{~nm}$ by a constant, is shown in Fig. 2(d) by a dashed line. While the energies are shifted the lines have almost the same width.

The full absorption spectrum of a CdSe QD is shown in Fig. 3. As we have in sets (B) and (C) different Gauss

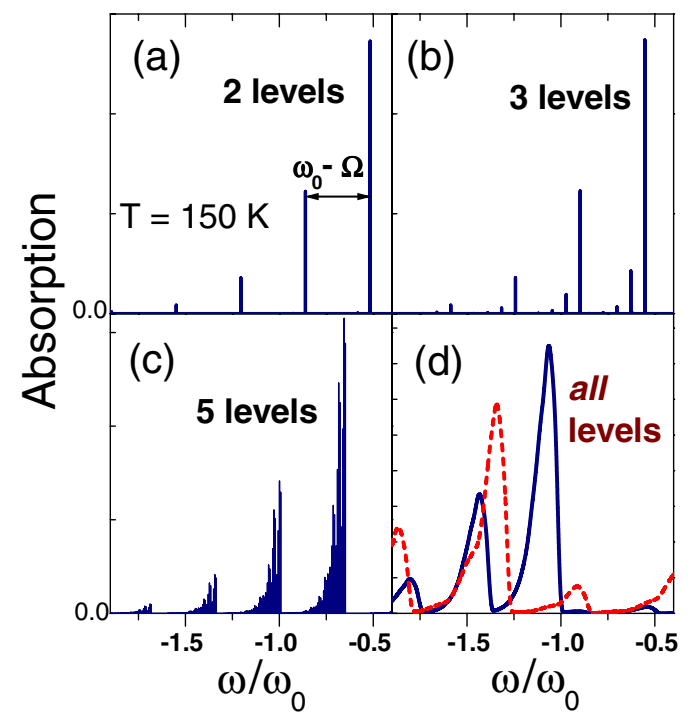

FIG. 2 (color online). Evolution of the fine structure in the absorption spectrum of a QD by accounting for more and more excitonic levels in parabolic potentials [linear scale, set (A), $E_{1}$ is taken as zero of energy]. The dashed curve in (d) shows the spectrum of a $5 \mathrm{~nm}$ QD having only one localized (discrete) exciton state and all the others delocalized (wetting-layer continuum). 


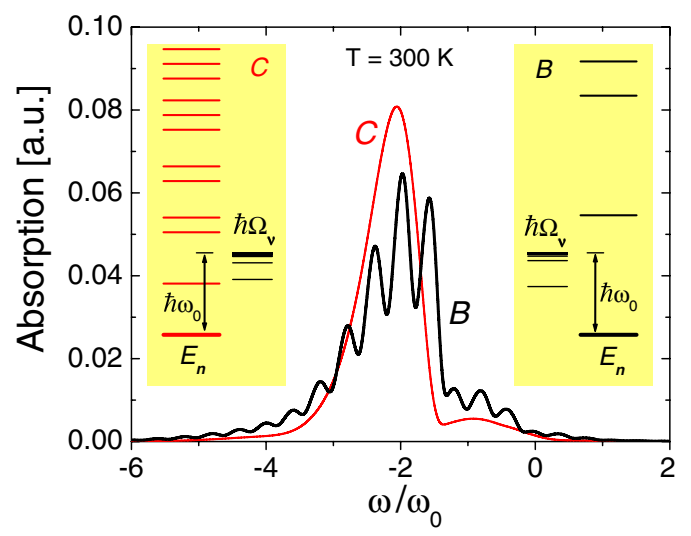

FIG. 3 (color online). Absorption spectrum of a CdSe QD at $T=300 \mathrm{~K}$, calculated for two different types of the bareexciton level structure [sets (B) and (C), see text]. Bound-phonon frequencies $\Omega_{\nu}$ and levels $E_{n}$ of exciton excited (ground) states are shown in the insets by thin (thick) horizontal lines.

lengths, $l_{e} \neq l_{h}$, the linear coupling $V_{L}$ contributes too, which gives surprisingly only a slight modification in the spectrum. For set (B) all the excited levels are well above $\hbar \omega_{0}$. All the bound-phonon frequencies $\Omega_{\nu}$ are condensed just below $\omega_{0}$, except one which is well separated and is in fact responsible for the spectral fine structure. Such an accumulation of infinitely many bound-phonon levels is a mathematical clue for the spectral broadening. The fine structure is absent if we take a larger dot with closer levels [Fig. 3, gray (red) curve and inset C]. Here, one excited level is found below $\hbar \omega_{0}$, and even two bound phonons are well resolved and wash out the fine structure. Thus, changes in the exciton level structure modify the spectrum but obviously do not affect our principal conclusion on the finite spectral width, and even do not alter much the linewidth itself.

Since the decay is not strictly exponential (see the inset of Fig. 4), we have extracted the linewidth directly from the absorption and plotted in Fig. 4 the full width at half

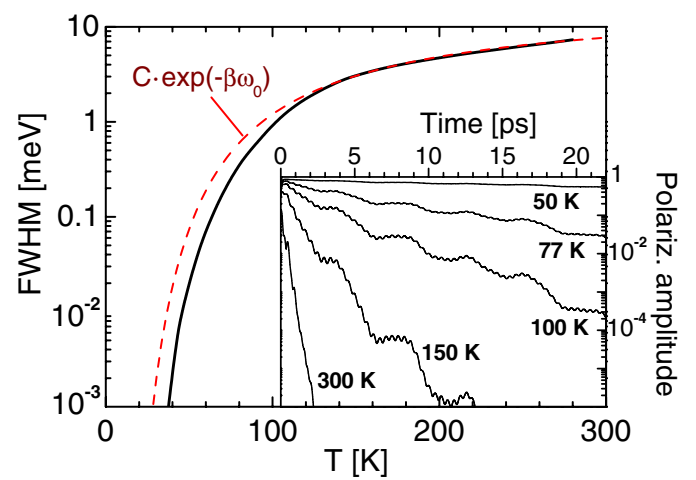

FIG. 4 (color online). Spectral linewidth of the ground exciton state in a CdSe QD, set (B), as a function of temperature (solid curve) and the exponential fit with $C=20 \mathrm{meV}$ (dashed curve). Inset: Polarization amplitude at different temperatures. maximum (FWHM) as a function of temperature. At high temperatures, the FWHM varies from a few to tens of milli-electron volts and can be well approximated by an exponential law with $\hbar \omega_{0}$ being the activation energy. Such activated behavior is in qualitative agreement with linewidth measurements in CdSe QDs [18] and nanocrystals [19]. Below $50 \mathrm{~K}$ the measured linewidth is dominated by coupling to acoustic phonons [4], phonon anharmonicity [20], and radiative decay.

In conclusion, we have calculated the time-dependent optical polarization and the absorption spectrum of a quantum dot linearly coupled to LO phonons by mapping this problem into an exactly solvable quadratic coupling model. Extending this model to an arbitrary number of excitonic states in a quantum dot, we have demonstrated that only accounting for a sufficient number of states results in a LO phonon-induced exciton dephasing and spectral broadening.

Financial support by DFG Sonderforschungsbereich 296 is gratefully acknowledged. E. A. M. acknowledges partial support by the Russian Foundation for Basic Research and the Russian Academy of Sciences.

*Electronic address: muljarov@gpi.ru

[1] U. Bockelmann and G. Bastard, Phys. Rev. B 42, 8947 (1990).

[2] P. Borri et al., Phys. Rev. Lett. 87, 157401 (2001).

[3] U. Bockelmann, Phys. Rev. B 48, 17637 (1993).

[4] E. A. Muljarov and R. Zimmermann, Phys. Rev. Lett. 93, 237401 (2004).

[5] S. Hameau et al., Phys. Rev. Lett. 83, 4152 (1999).

[6] T. Stauber, R. Zimmermann, and H. Castella, Phys. Rev. B 62, 7336 (2000).

[7] O. Verzelen, R. Ferreira, and G. Bastard, Phys. Rev. Lett. 88, 146803 (2002).

[8] L. Jacak et al., Phys. Rev. B 67, 035303 (2003).

[9] T. Stauber and R. Zimmermann, Phys. Rev. B 73, 115303 (2006).

[10] T. Inoshita and H. Sakaki, Phys. Rev. B 56, R4355 (1997).

[11] K. Král and Z. Khás, Phys. Rev. B 57, R2061 (1998).

[12] A. V. Uskov et al., Phys. Rev. Lett. 85, 1516 (2000).

[13] E. A. Muljarov and R. Zimmermann, Phys. Rev. Lett. 96, 019703 (2006).

[14] P. J. Dean, D. D. Manchon, Jr., and J. J. Hopfield, Phys. Rev. Lett. 25, 1027 (1970).

[15] L. Jacak et al., Eur. Phys. J. D 22, 319 (2003).

[16] J. Seebeck et al., Phys. Rev. B 71, 125327 (2005).

[17] Electron (hole) energy levels are given by $(2 n+l) \Delta_{e(h)}$; 30 levels are produced by taking $0 \leq n \leq 4,0 \leq l \leq 5$.

[18] F. Gindele et al., Phys. Rev. B 60, R2157 (1999); J. Seufert et al., Phys. Rev. B 62, 12609 (2000); K. Sebald et al., Appl. Phys. Lett. 81, 2920 (2002); A. M. Kapitonov et al., Phys. Rev. B 70, 195304 (2004).

[19] S. Nomura and T. Kobayashi, Phys. Rev. B 45, 1305 (1992); D. M. Mittleman et al., Phys. Rev. B 49, 14435 (1994).

[20] P. Machnikowski, Phys. Rev. Lett. 96, 140405 (2006). 